\title{
METRIC-DEPENDENT DIMENSION FUNCTIONS ${ }^{1}$
}

\section{K. NAGAMI AND J. H. ROBERTS}

The starting point of this study was an attempt to solve a problem proposed by G. M. Rosenstein [3] in his doctoral dissertation.

RosensteIn's PROBLEM. Suppose that $R$ is a metrizable space, $\operatorname{dim} R \geqq n$ (covering dimension), and $\rho$ is any compatible metric for $R$. Then is it true that there exist $n$ pairs of closed sets $C_{1}, C_{1}^{\prime}, \cdots$, $C_{n}, C_{n}^{\prime}$ such that (i) $\rho\left(C_{i}, C_{i}^{\prime}\right)>0$ for all $i$ and (ii) if for each $i, B_{i}$ is a closed set separating $C_{i}$ and $C_{i}^{\prime}$, then $\bigcap_{i=1}^{n} B_{i} \neq \varnothing$ ?

REMARK. If, in this problem, "(i) $\rho\left(C_{i}, C_{i}^{\prime}\right)>0$ " is replaced by "(i*) $C_{i} \cap C_{i}^{\prime}=\varnothing$," one obtains a characteristic property for metric spaces $R$ such that $\operatorname{dim} R \geqq n$. (See [1, Remark, for the separable case, p. 78].)

Our main result is an example which shows that the answer to the problem is in the negative. In the notation given below, our example is a metric space $(R, \rho)$ such that $\operatorname{dim} R=2$ and $d_{2}(R, \rho)=1$. For $n=1$ the problem is answered in the affirmative.

Definition of $d_{1}(R, \rho)$. Let $(R, \rho)$ be a metric space with metric $\rho$. We define $d_{1}(R, \rho)$ inductively as follows: For the empty set $\varnothing$, $d_{1}(\varnothing, \rho)=-1$. If for every pair of closed subsets $F, H$ of $R$ with $\rho(F, H)>0$ there exists an open set $G$ with $F \subset G \subset R-H$ and with $d_{1}\left(\bar{G}-G, \rho^{*}\right) \leqq n-1$, where $\rho^{*}$ is the restriction of $\rho$ to $\bar{G}-G$, then we say $d_{1}(R, \rho) \leqq n$. If there is no such integer $n$, then we say $d_{1}(R, \rho)=\infty$.

TheOREM. For any metric space $(R, \rho)$ we have $d_{1}(R, \rho)=$ Ind $R$, where Ind $R$ is the large inductive dimension of $R$ defined by means of sets separating disjoint closed pairs of subsets.

Proof. It is evident that $d_{1}(R, \rho) \leqq$ Ind $R$. When $d_{1}(R, \rho)=\infty$, it is also evident that $d_{1}(R, \rho) \geqq$ Ind $R$. Hence we suppose that $d_{1}(R, \rho)$ $\leqq n$ and make the induction assumption that $d_{1}\left(R^{\prime}, \rho^{\prime}\right) \geqq$ Ind $R^{\prime}$ for any metric space $\left(R^{\prime}, \rho^{\prime}\right)$ with $d_{1}\left(R^{\prime}, \rho^{\prime}\right) \leqq n-1$. Let $H$ and $F$ be disjointclosed subsets of $R$. Put $D_{i}=\{x: \rho(x, H)<1 / i\}$, and $E_{i}=\{x: \rho(x, F)$ $<1 / i\}, i=1,2, \cdots$. Then there exist open sets $M_{i}$ with $H \subset M_{i}$ $C D_{i}, d_{1}\left(\bar{M}_{i}-M_{i}, \rho\right) \leqq n-1, i=1,2, \cdots$, and open sets $N_{i}$ with $F \subset N_{i} \subset E_{i}, d_{1}\left(\bar{N}_{i}-N_{i}, \rho\right) \leqq n-1, i=1,2, \cdots$. Put $G_{i}=M_{i}-\bar{N}_{i}$, $i=1,2, \cdots$, and $G=\bigcup_{i=1}^{\infty} G_{i}$. Then it can easily be seen that $H \subset G$ $\subset \bar{G} \subset R-F$.

Presented to the Society, August 25, 1965; received by the editors May 6, 1964.

1 This research was supported in part by the National Science Foundation, Grant GF-2065. 
Since $\bar{G}_{i}-G_{i} \subset\left(\bar{M}_{i}-M_{i}\right) \cup\left(\bar{N}_{i}-N_{i}\right)$, we have Ind $\left(\bar{G}_{i}-G_{i}\right) \leqq n-1$ by the induction assumption. Since $\left\{G_{i}: i=1,2, \cdots\right\}$ is locally finite at every point of $R-(H \cup F)$ and $\bar{G}-G$ is contained in $R-(H \cup F)$, we have $\bar{G}-G \subset \cup\left\{\bar{G}_{i}-G_{i}: i=1,2, \cdots\right\}$. Every point $x$ of $\bar{G}-G$ has a relative neighborhood $U(x)$ which is contained in the sum of a finite number of elements of $\left\{\bar{G}_{i}-G_{i}: i=1,2, \cdots\right\}$. Hence Ind $U(x) \leqq n-1$ and we have $\operatorname{Ind}(\bar{G}-G) \leqq n-1$ by the local dimension theorem. Thus we know Ind $R \leqq n$ and the theorem is proved.

CoRollary. If $R$ is a metrizable space with $\operatorname{dim} R=1$ and $\rho$ is any compatible metric for $R$, then there exists a pair of closed sets $C$ and $C^{\prime}$ with $\rho\left(C, C^{\prime}\right)>0$ such that the empty set cannot separate $C$ and $C^{\prime}$.

Definition of $d_{2}(R, \rho)$. Let $(R, \rho)$ be a metric space with metric $\rho$. We write $d_{2}(\varnothing, \rho)=-1$. If there exists a greatest integer $n$ such that there exist $n$ pairs $C_{1}, C_{1}^{\prime}, \cdots, C_{n}, C_{n}^{\prime}$ such that (i) $\rho\left(C_{i}, C_{i}^{\prime}\right)>0$ and (ii) if for each $i, B_{i}$ is a closed set separating $R$ between $C_{i}$ and $C_{i}^{\prime}$, then $\bigcap_{i=1}^{n} B_{i} \neq \varnothing$, then we say $d_{2}(R, \rho)=n$. Otherwise $d_{2}(R, \rho)$ $=\infty$.

Rosenstein's problem may now be stated as follows: Is it true that $d_{2}(R, \rho)=\operatorname{dim} R$ ?

EXAMPLE. In the closed 3 -cell $I^{3}$ we define a countable disjoint collection $a=\left\{A_{i}: i=1,2, \cdots\right\}$, set $A=\cup Q$ and $R=I^{3}-A$. We prove that $\operatorname{dim} R=2$ and $d_{2}(R, \rho)=1$, where $\rho$ is the Euclidean metric on $I^{3}$. Let $u=\left\{U_{1}, U_{2}, \cdots\right\}$ be any countable base for the topology of $I^{3}$. The set of all finite unions of elements of $\mathcal{U}$ is countable; hence so is the set of all quadruples of such unions and, a fortiori, any subset. Thus there exists a countable sequence $Q_{1}, Q_{2}, \cdots$, such that for all $i$

(1) $Q_{i}=\left\{B_{i}, C_{i}, D_{i}, E_{i}\right\}$,

(2) each of $B_{i}, C_{i}, D_{i}, E_{i}$ is a finite union of elements of $\mathcal{u}$,

(3) $\rho\left(B_{i}, C_{i}\right)>0$ and $\rho\left(D_{i}, E_{i}\right)>0$, and

(4) if $Q^{\prime}=\left\{B^{\prime}, C^{\prime}, D^{\prime}, E^{\prime}\right\}$ is any quadruple satisfying (2) and (3) then for some $i$ we have $Q^{\prime}=Q_{i}$.

For each $i$ we define closed sets $M_{i}$ and $N_{i}$ such that $M_{i}$ separates $B_{i}$ and $C_{i}, N_{i}$ separates $D_{i}$ and $E_{i}$, and we set $A_{i}=M_{i} \cap N_{i}$. We want $a=\left\{A_{i}: i=1,2, \cdots\right\}$ to be a disjoint collection. Let $\epsilon_{i}$ $=\min \left\{\rho\left(B_{i}, C_{i}\right), \rho\left(D_{i}, E_{i}\right)\right\}$. Let $\pi_{1}, \pi_{1}^{\prime}, \pi_{2}, \pi_{2}^{\prime}, \cdots$ be a monotonically increasing sequence of prime numbers such that for all $i$, $1 / \pi_{i}<\epsilon_{i} / \sqrt{ } 3$. Divide $I^{3}$ into $\pi_{i}^{3}$ small closed cubes whose edges have length $1 / \pi_{i}$, let $H_{i}$ denote the union of all such cubes that intersect $B_{i}$, and let $M_{i}=$ boundary of $H_{i}$. Similarly, using $\pi_{i}^{\prime}$ instead of $\pi_{i}$, 
let $K_{i}$ be the union of all small cubes intersecting $D_{i}$ and let $N_{i}$ = boundary of $K_{i}$. Set $A_{i}=M_{i} \cap N_{i}$. If $x \in A_{i}$, then one coordinate of $x$ is of the form $a / \pi_{i}$ and one coordinate is of the form $b / \pi_{i}^{\prime}$, where $a$ and $b$ are integers, $0<a<\pi_{i}, 0<b<\pi_{i}^{\prime}$.

Assertion 1 . For all $i, M_{i}$ is a closed set separating $B_{i}$ and $C_{i}$ and $N_{i}$ is a closed set separating $D_{i}$ and $E_{i}$.

Assertion 2. For all $i, A_{i}$ is closed and $\operatorname{dim} A_{i} \leqq 1$.

Proof. If $a$ and $b$ are positive integers with $0<a<\pi_{i}, 0<b<\pi_{i}^{\prime}$, then $a / \pi_{i} \neq b / \pi_{i}^{\prime}$; hence if $x \in A_{i}$, then at least two of its coordinates are rational. By $\left[1\right.$, Example III 6, p. 29] this shows that $\operatorname{dim} A_{i} \leqq 1$.

Assertion 3. If $i \neq j$, then $A_{i} \cap A_{j}=\varnothing$.

Proof. Assume there exists $x \in A_{i} \cap A_{j}$. Then there exist four integers $0<a<\pi_{i}, 0<b<\pi_{i}^{\prime}, 0<c<\pi_{j}, 0<d<\pi_{j}^{\prime}$ such that $x$ must have coordinates equal to each of $a / \pi_{i}, b / \pi_{i}^{\prime}, c / \pi_{j}, d / \pi_{j}^{\prime}$, four distinct numbers. But $x$ has only 3 coordinates.

ASSERTION 4. $d_{2}(R, \rho) \leqq 1$.

Proof. Let $B, C, D, E$ be any relatively closed sets in $R$ such that $\rho(B, C)>0, \rho(D, E)>0$. Then their closures in $I^{3}$ are compact and for some $i$ we have $B \subset B_{i}, C \subset C_{i}, D \subset D_{i}$ and $E \subset E_{i}$; so $M_{i}$ separates $B$ and $C$ in $I^{3}, N_{\text {s }}$ separates $D$ and $E$ in $I^{3}$. But then $M_{i} \cap R$ and $N_{i} \cap R$ are corresponding separating sets in $R$, and their intersection is vacuous since $M_{i} \cap N_{i}=A_{i} \subset I^{3}-R$.

Assertion 5. $\operatorname{dim} R \leqq 2$.

Proof. This follows from Brouwer's theorem on invariance of domain, since it can easily be seen that $A$ is dense in $I^{3}$.

Assertion $6 . \operatorname{dim} R \geqq 2$.

To prove this assertion we need the following two lemmas.

LemMA 1. Let $S$ be a subset of the closed $n$-cell $I^{n} w i t h \operatorname{dim} S \leqq n-2$. Then for any points $p$ and $q$ in $I^{n}-S$ there exists a continuum $K$ such that (i) $K$ is contained in $I^{n}-S$ and (ii) $K$ contains $p$ and $q$. (See [4].)

Lemma 2. A continuum cannot be decomposed into a countably infinite or finite (but more than one) union of pairwise disjoint closed subsets. (See [2, Theorem 44, p. 30].)

Proof of assertion 6. Assume that $\operatorname{dim} R<2$. If $A$ is closed, then $R$ is an $F_{\sigma}$ and $\operatorname{dim} I^{3} \leqq \max (\operatorname{dim} A, \operatorname{dim} R)<2$, which is impossible. Thus there exist integers $i$ and $j$ with $i \neq j$ such that $A_{i} \neq \varnothing, A_{j} \neq \varnothing$. Take $p \in A_{i}, q \in A_{j}$. By Lemma 1 there exists a continuum $K$ such that (i) $\{p, q\} \subset K$ and (ii) $K \subset I^{3}-R=A$. Thus $K=\bigcup_{i=1}^{\infty}\left(K \cap A_{i}\right)$, a countable union of pairwise disjoint closed sets at least two of which 
are not vacuous. But this is impossible by Lemma 2, so the proof of Assertion 6 is completed.

Assertion 7. $d_{2}(R, \rho) \geqq 1$.

Proof. Since $\operatorname{dim} R=2, d_{2}(R, \rho) \neq-1$. It is obvious that $d_{2}(R, \rho)$ $=0$ if and only if $d_{1}(R, \rho)=0$. On the other hand we already know that $d_{1}(R, \rho)=\operatorname{dim} R$ by the above theorem. Thus $d_{2}(R, \rho)<1$ contradicts $\operatorname{dim} R=2$.

REMARK. By a construction quite similar to that just given, one may start with $I^{n}$ for $n>3$, and define a subset $R$ such that $\operatorname{dim} R$ $=n-1$ and $d_{2}(R, \rho) \leqq n / 2$.

Problem. Is it true that, given a metric space $(R, \rho), \operatorname{dim} R \geqq n \mathrm{im}$ plies that $d_{2}(R, \rho) \geqq n / 2$ ?

\section{REFERENCES}

1. W. Hurewicz and H. Wallman, Dimension theory, Princeton Mathematical Series, Vol. 4, Princeton Univ. Press, Princeton, N. J., 1941.

2. R. L. Moore, Foundations of point set theory, Amer. Math. Soc. Colloq. Publ. Vol. 13, Amer. Math. Soc., Providence, R. I., 1932.

3. G. M. Rosenstein, Jr., A further extension of Lebesgue's covering theorem, Proc. Amer. Math. Soc. 15 (1964), 683-688.

4. K. Sitnikov, On the dimension of non-closed sets of Euclidean space, Dokl. Akad. Nauk SSSR 83 (1952), 31-34. (Russian)

DUKE UNIVERSITY 